\title{
АНАЛИЗ ПРИМЕНЕНИЯ ПАРАМЕТРИЧЕСКОГО И НЕПАРАМЕТРИЧЕСКОГО МЕТОДОВ ОБРАБОТКИ НА ПРИМЕРЕ ДАННЫХ ЗНАЧЕНИЙ СИЛЫ ТЯЖЕСТИ
}

\author{
Наталья Николаевна Кобелева \\ Сибирский государственный университет геосистем и технологий, 630108, Россия, г. Новоси- \\ бирск, ул. Плахотного, 10, кандидат технических наук, доцент кафедры космической и физи- \\ ческой геодезии, тел. (383)361-01-59, e-mail: n.n.kobeleva@mail.ru
}

\section{Зарина Баранкуловна Бексултанова}

Коммунальное государственное казенное предприятие «Высший колледж геодезии и картографии» управления образования Восточно-Казахстанской области, 071400, Казахстан, г. Семей, ул. Заря, 42, бакалавр геодезии и картографии, преподаватель специальных дисциплин, тел. (778)470-74-62, e-mail: beksultanova_zarina90@mail.ru

В статье рассматриваются результаты исследования применения непараметрической обработки геодезических измерениий. Выполнен сравнительный анализ параметрической и непараметрической оценки с результатами геодезических измерений на примере определения силы тяжести. Для реализации данного исследования использовалось программное обеспечение Statgraphics 18 и мастер диаграмм Microsoft Excel. По результам измерений вычислены такие характеристики как медиана, среднее арифметическое, стандартное отклонение и дисперсия, а также получены значения доверительных интервалов для измерений силы тяжести в расчете двухсот семидесяти отсчетов с сейсмозащитой и без сейсмозащиты. Приведены разности между полученной шириной доверительных интервалов для двух приемов, построены диаграммы, проведен анализ полученных результатов.

Ключевые слова: непараметрический и параметрический анализ, доверительные интервалы, медиана, среднее арифметическое, дисперсия, робастные методы, выборка, гистограмма, стандартное отклонение, сила тяжести, сейсмозащита, нормальный закон распределения, зашумление, состоятельность, несмещенность и эффективность

\section{ANALYSIS OF THE APPLICATION OF PARAMETRIC AND NONPARAMETRIC PROCESSING METHODS ON THE EXAMPLE OF THESE VALUES OF GRAVITY}

\section{Natalia N. Kobeleva}

Siberian State University of Geosystems and Technologies, 10, Plakhotnogo St., Novosibirsk, 630108, Russia, Ph. D., Associate Professor, Department of Space and Physical Geodesy, phone: (383)361-01-59, e-mail: n.n.kobeleva@mail.ru

\section{Zarina B. Beksultanova}

State Utility Company "Higher College of Geodesy and Cartography" of the Education Department of the East Kazakhstan Region, 42, Zarya St., Semey, 071400, Kazakhstan, Bachelor of Geodesy and Cartography, Teacher of Special Disciplines, phone: (778)470-74-62, e-mail: beksultanova_zarina90@mail.ru

The article discusses the results of studying the application of nonparametric processing of geodetic measurements. A comparative analysis of parametric and nonparametric estimates to the results of geodetic measurements is carried out using the example of determining the force of gravity. To implement the study, Statgraphics 18 software and Microsoft Excel chart wizard were used. According to the measurement results, such characteristics as the median, arithmetic mean, standard 
deviation and variance were calculated, as well as the values of the confidence intervals for gravity measurements in the calculation of two hundred and seventy readings with and without seismic protection were obtained. Differences between the obtained width of the confidence intervals for the two methods are given, diagrams are constructed, and the analysis of the obtained results obtained is carried out.

Keywords: nonparametric and parametric analysis, confidence intervals, median, arithmetic mean, variance, robust methods, sample, histogram, standard deviation, gravity, seismic protection, normal distribution, noise, consistency, unbiasedness and efficiency, observations

\section{Введение}

Методы обработки как экспериментальных, так и натурных данных направлены, прежде всего, на анализ, оценку и на уменьшение влияния погрешностей результатов измерений [1]. В связи с этим, на сегодняшний день статистические методы обработки данных являются достаточно разработанными, распространенными и широко применяются на практике. При этом они подразделяются на три основные группы: классические (традиционные), робастные и непараметрические.

Классические методы - это параметрические методы, построенные на гипотезе нормального распределения исследуемых данных, анализе и оценке этих параметров распределения [2].

Робастные методы ориентированны на нахождение выбросов из всей совокупности измерений, уменьшения их воздействия или удаления их из выборки [3].

Непараметрические методы - это методы, которые не зависят от какихлибо параметров, и дают возможность обрабатывать результаты наблюдений «низкого качества», о распределении которых не так много информации [4].

Классические и робастные методы обработки данных, как правило, чаще всего используют в качестве оценки центра распределения - среднее арифметическое результатов наблюдений. Робастные методы более устойчивы к выбросам и шумам, чем классические, но все же они остаются параметрическими. В отличие от них непараметрические методы не связаны с какими-либо определенными параметрами, а в качестве центра распределения принимают медиану, а не математическое ожидание искомой выборки.

\section{Исходные данные}

Ускорения силы тяжести были получены по договоренности с геодезической экспедицией №145, города Новосибирск. Наблюдения выполнялись двумя приемами динамическим методом [5]: в первом приеме на приборе ГБЛ (гравиметр баллистический лазерный) была установлена сейсмозащита, исключающая влияние на отсчеты внешних воздействий, во втором приеме сейсмозащита отсутствовала. Значения силы тяжести получены в условной системе, размерность измерения - мили Галлы. 
В выполненных приемах, производили по три цикла наблюдений, по девяносто отсчетов в каждом цикле. Измерения производились в соответствии с требованиями инструкции [6].

\section{Исследования}

В астрономо-геодезических наблюдениях, выполняя многократные измерения одной и той же величины, при одинаковых условиях, полученные результаты отличаются друг от друга на некоторую величину, то есть поддаются случайному рассеиванию [7].

Колебание полученных отсчетов ускорения силы тяжести, как массовые повторные измерения, поддаются математической закономерности распределения [8]. Для прослеживания распределения полученных результатов силы тяжести были построены гистограммы, для двух приемов (рис.1 и 2).

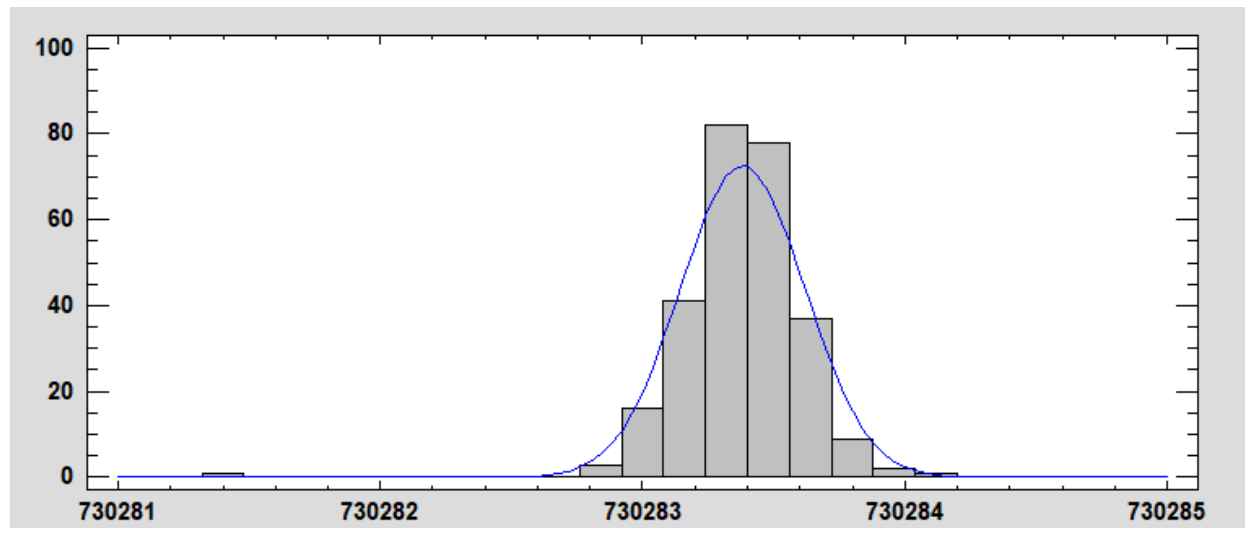

Рис. 1. Гистограмма отсчетов с сейсмозащитой

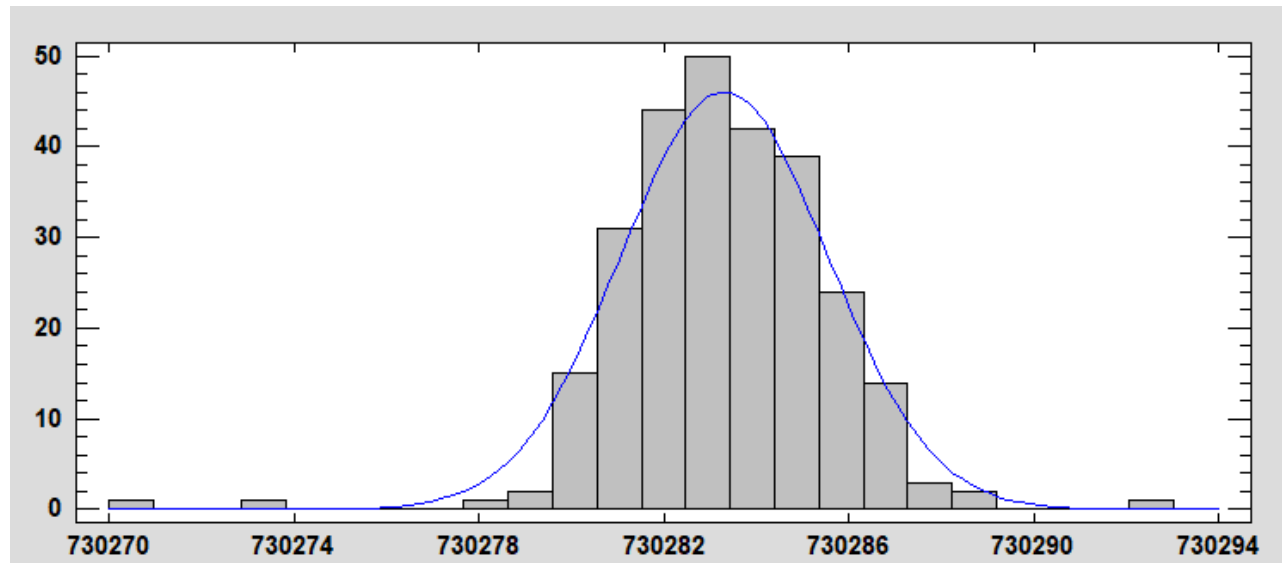

Рис. 2. Гистограмма отсчетов без сейсмозащиты

Можно отметить, что с увеличением количества измерений, мы наблюдается размещение полученных результатов ускорений силы тяжести, соответсвующие нормальному закону распределения [7]. 
Обработка полученных ускорений силы тяжести будет выполняться с использованием оценки математической статистики [9]. Суть исследования будет состоять в нахождении параметрической и непараметрической оценки полученных результатов измерения силы тяжести и сравнения полученной точности.

В табл. 1 и 2 приведены среднеарифметическая и медианная оценки измерений силы тяжести, сравнение выполнялось по всем трем циклам каждого приема: с сейсмозащитой и без.

Для анализа рассматриваемых оценок были найдены такие характеристики, как среднее арифметическое, медиана, стандартное отклонение и дисперсия [10-13].

Таблица 1

Статистические показатели оценок значений силы тяжести, полученных с сейсмозащитой

\begin{tabular}{|c|c|c|c|}
\hline Характеристики & 1 серия & 2 серия & 3 серия \\
\hline $\begin{array}{l}\text { Среднее } \\
\text { арифметическое }\end{array}$ & 730283,4038 & 730283,3884 & 730283,2131 \\
\hline Медиана & 730283,4170 & 730283,3533 & 730283,3335 \\
\hline Дисперсия по среднему арифметическому & 0,03923 & 0,05479 & 0,18485 \\
\hline Дисперсия по медиане & 0,03897 & 0,05541 & 0,19730 \\
\hline $\begin{array}{l}\text { Стандартное отклонение по среднему арифме- } \\
\text { тическому }\end{array}$ & 0,19806 & 0,23406 & 0,42995 \\
\hline Стандартное отклонение по медиане & 0,19740 & 0,23539 & 0,44418 \\
\hline \multirow{2}{*}{$\begin{array}{l}\text { Границы доверительного } \\
\text { интервала по среднему арифметическому }\end{array}$} & 730283,0156 & 730282,9296 & 730282,3704 \\
\hline & 730283,7920 & 730283,8471 & 730284,0558 \\
\hline \multirow{2}{*}{$\begin{array}{l}\text { Границы доверительного } \\
\text { интервала по медиане }\end{array}$} & 730283,0301 & 730282,8919 & 730282,4629 \\
\hline & 730283,8038 & 730283,8146 & 730284,2041 \\
\hline \multirow{2}{*}{$\begin{array}{l}\text { Границы доверительного } \\
\text { интервала по распределению Стьюдента для } \\
\text { среднего арифметического }\end{array}$} & 730283,0102 & 730282,9233 & 730282,3588 \\
\hline & 730283,7973 & 730283,8534 & 730284,0674 \\
\hline \multirow{2}{*}{$\begin{array}{l}\text { Границы доверительного } \\
\text { интервала по распределению Стьюдента для } \\
\text { медианы }\end{array}$} & 730283,0247 & 730282,8855 & 730282,4509 \\
\hline & 730283,8092 & 730283,8210 & 730284,2161 \\
\hline
\end{tabular}

Вычисленные числовые характеристики (табл.1, табл.2) не дают полной информации о точности и надежности полученной оценки [14]. Показатель точечного оценивания устанавливается такими характеристиками как состоятельность, несмещенность и эффективность [15].

Несмещенность оценки означает, что ее математическое ожидание равно значению оцениваемого параметра генеральной совокупности [16]. Для сравнения меры эффективности $e$ точечного оценивания пользуются следующим соотношением: 


$$
e=\frac{M \times\left(\theta_{1}-\theta\right)^{2}}{M \times\left(\theta_{2}-\theta\right)^{2}},
$$

где $\theta_{1}$ и $\theta_{2}$ - оценки, характеризующие параметр $\theta, M$ - математическое ожидание.

При $e>1$, оценка $\theta_{2}$ считается эффективнее, чем $\theta_{1}$ (и обратно), потому что она располагает меньшим рассеиванием.

Таблица 2

Статистические показатели оценок значений силы тяжести, полученных без сейсмозащиты

\begin{tabular}{|c|c|c|c|}
\hline Характеристики: & 1 серия & 2 серия & 3 серия \\
\hline $\begin{array}{l}\text { Среднее } \\
\text { арифметическое }\end{array}$ & 730283,5793 & 730283,2921 & 730283,0226 \\
\hline Медиана & 730283,3891 & 730283,2545 & 730282,9946 \\
\hline Дисперсия по среднему арифметическому & 4,25768 & 4,91534 & 5,52704 \\
\hline Дисперсия по медиане & 4,24656 & & 5,46642 \\
\hline $\begin{array}{l}\text { Стандартное отклонение по среднему ариф- } \\
\text { метическому }\end{array}$ & 2,06342 & 2,21706 & 2,35097 \\
\hline Стандартное отклонение по медиане & 2,06072 & 2,20503 & 2,33804 \\
\hline \multirow{2}{*}{$\begin{array}{l}\text { Границы доверительного } \\
\text { интервала по среднему арифметическому }\end{array}$} & 730279,5350 & 730278,9467 & 730278,4147 \\
\hline & 730287,6236 & 730287,6376 & 730287,6305 \\
\hline \multirow{2}{*}{$\begin{array}{l}\text { Границы доверительного } \\
\text { интервала по медиане }\end{array}$} & 730279,3500 & 730278,9326 & 730278,4120 \\
\hline & 730287,4281 & 730287,5763 & 730287,5771 \\
\hline \multirow{2}{*}{$\begin{array}{l}\text { Границы доверительного } \\
\text { интервала по распределению Стьюдента для } \\
\text { среднего арифметического }\end{array}$} & 730279,4793 & 730278,8869 & 730278,3512 \\
\hline & 730287,6792 & 730287,6974 & 730287,6939 \\
\hline \multirow{2}{*}{$\begin{array}{l}\text { Границы доверительного } \\
\text { интервала по распределению Стьюдента для } \\
\text { медианы }\end{array}$} & 730279,2944 & 730278,8731 & 730278,3489 \\
\hline & 730287,4837 & 730287,6358 & 730287,6402 \\
\hline
\end{tabular}

Эффективность оценки так же можно определить соотношением дисперсий, если $\theta_{1}$ и $\theta_{2}$ - несмещенные оценки:

$$
e=\frac{\sigma^{2} \theta_{1}}{\sigma^{2} \theta_{2}}
$$

a $\sigma^{2} \theta_{1}$ и $\sigma^{2} \theta_{2}-$ дисперсии соответствующих оценок.

Из формулы (2) следует - чем меньше дисперсия тем эффективнее оценка.

Состоятельность оценки определяется постоянством несмещенности оценки, при безграничных объемах выборки [7].

По результатам исследований в табл. 2 можно заметить, что значение дисперсии при непараметрическом оценивании является минимальным, исходя из 
этого можно сделать выводы о том, что выполняется требование несмещенности и эффективности оценки [17].

Помимо точечного оценивания используется и интервальное. Любая статистическая оценка параметра, выполненная по полученным данным наблюдений, становится объективнее при определении границ допустимых погрешностей [7].

При сжатом числе наблюдений оценку точности рекомендовано проводить посредством нахождения доверительных интервалов [18, 19].

Построение доверительных интервалов выполнялось по средней арифметической и медианой оценкам, по формулам (3) и (4) [13].

Доверительный интервал для математического ожидания при известной дисперсии $\sigma$ :

$$
\bar{x}-\varepsilon_{\beta} \times\left(\frac{\sigma}{\sqrt{n}}\right)\left\langle\alpha \left\langle\bar{x}+\varepsilon_{\beta} \times\left(\frac{\sigma}{\sqrt{n}}\right),\right.\right.
$$

где n- объем выборки, ${ }^{\varepsilon_{\beta}}$ - значение аргумента функции Лапласа, $\bar{x} \cdot$ - выборочное среднее. И если не известна дисперсия, то используется следующая формула:

$$
\bar{x}-t_{\beta} \times\left(\frac{s}{\sqrt{n}}\right)\left\langle\alpha \left\langle\bar{x}+t_{\beta} \times\left(\frac{s}{\sqrt{n}}\right),\right.\right.
$$

где $s$ - исправленное среднее квадратические отклонение, $t_{\beta}$ - значение аргумента функции распределения Стьюдента [20].

Анализируя результаты вычислений (табл. 1, табл. 2), можно сделать вывод, что если в серии наблюдений присутствуют выбросы (шум), то медианная оценка дает более точный результат. Об этом можно судить и по границам доверительных интервалов, чем они уже, тем точнее оценка. Сравнение значений границ доверительных интервалов приведены в табл. 3.

Таблица 3

Сравнение значений ширины доверительных интервалов двух приемов

\begin{tabular}{|l|c|c|c|c|c|c|}
\hline \multirow{2}{*}{$\begin{array}{c}\text { нахождения доверительного } \\
\text { интервала }\end{array}$} & \multicolumn{2}{|c}{$\begin{array}{c}\text { Ширина доверительных ин- } \\
\text { тервалов с } \\
\text { сейсмозащитой }\end{array}$} & \multicolumn{2}{|c|}{$\begin{array}{c}\text { Ширина доверительных } \\
\text { интервалов без } \\
\text { сейсмозащиты }\end{array}$} \\
\cline { 2 - 8 } & 1 & 2 & 3 & 1 & 2 & 3 \\
\hline $\begin{array}{l}\text { По среднему арифметиче- } \\
\text { скому }\end{array}$ & 0,7764 & 0,9175 & 1,6854 & 8,0886 & 8,6909 & 9,2158 \\
\hline По медиане & 0,7738 & 0,9227 & 1,7412 & 8,0780 & 8,6437 & 9,1651 \\
\hline $\begin{array}{l}\text { Разница доверительных интер- } \\
\text { валов }\end{array}$ & $-0,0026$ & $+0,0052$ & $+0,0561$ & $-0,0106$ & $-0,472$ & $-0,0507$ \\
\hline $\begin{array}{l}\text { По распределению Стьюдента } \\
\text { для среднего } \\
\text { арифметического }\end{array}$ & 0,7871 & 0,9302 & 1,7086 & 8,1999 & 8,8105 & 9,3426 \\
\hline $\begin{array}{l}\text { По распределению Стьюдента } \\
\text { для медианы }\end{array}$ & 0,7844 & 0,9354 & 1,7652 & 8,1892 & 8,7627 & 9,2913 \\
\hline $\begin{array}{l}\text { Разница доверительных интер- } \\
\text { валов }\end{array}$ & $-0,0027$ & $+0,0052$ & $+0,0566$ & $-0,0107$ & $-0,0478$ & $-0,0513$ \\
\hline
\end{tabular}




\section{Заключение}

Выполняя анализ полученных исследований можно сделать следующие первоначальные выводы о применимости непараметрического метода к математической обработке геодезических данных:

1. По данным табл. 2, видно, что при точечном оценивании полученных наблюдений, выполняются такие требования как несмещенность и эффективность, о чем говорит постоянство минимальной дисперсии, если в качестве центра распределения принимать медиану данного вариационного ряда, а не среднеарифметическое значение;

2. При интервальной оценке, ширина доверительных интервалов уже по медианному оцениванию при наблюдениях, содержащих выбросы, т.е. в наблюдениях без сейсмозащиты. Это говорит о том, что среднеарифметическое оценивание лучше при наблюдениях, не содержащих выбросы.

\section{БИБЛИОГРАФИЧЕСКИЙ СПИСОК}

1. Нефедова Г.А., Ащеулов В.А. Теория вероятности и математическая статистика // учеб. пособие. - Новосибирск: СГГА, 2005. - 94 с.

2. Герасимов А.Н., Морозова Н.И. Параметрические и непараметрические методы в медицинской статистике // Эпидимиология и вакционопрофилактика №5 (84) /2015. C. 6-12.

3. Смирнов П.О. Робастные методы и алгоритмы оценивания корреляционныххарактеристик данных на основе новых высокоэффективных и быстрых робастных оценок масштаба // автореферат Санкт -Петербург - 2013. - С. 18.

4. Кобелева Н.Н. Разработка информационной технологии непараметрической обработки геодезических данных // Вестник Сибирской государственной геодезической академии. - Новосибирск, 2002. - Вып. 7. - С. 139-142.

5. Шароглазова Г.А. Гравиметрия // учеб. -метод. комплекс - Новополоцк: ПГУ, 2006 $196 \mathrm{c}$.

6. Инструкция по развитию высокоточной государственной гравиметрической сети России // М., ЦНИИГАиК 200166 с.

7. Смирнов Н.В., Белугин Д.А. Теория вроятностей и математическая статистика в приложении к геодезии, монография // М., «Недра», 1969, стр. 379.

8. Ламанова Н.Г. Статистическое оценивание результатов и погрешностей измерений при многократных наблюдениях // Перм.нац. ислед. политехн. ун-т. Пермь, 2017. 39с.

9. Луценко А.И. Математическая статистика // Ростов н/Д: Изд-во ЮФУ, 2014, 157c.

10. Балаш О.С. Методические указания и варианты контрольных заданий по статистике (часть 1) // Саратов $-46 \mathrm{c}$.

11. Гребенникова И.В. Методы математической обработки экспериментальных данных // уч.-методическое пособие Екатеринбург: Изд-во Урал. ун-та, 2015. - 124 с.

12. Спирин Н.А., Лавров В.В. Планирование эксперимента //Екатеринбург: Изд-во Урал. ун-та, 2015. - $124 \mathrm{c}$.

13. Лапко А. В., Лапко В. А. Непараметрические модели и алгоритмы обработки информации // учеб. пособие. - Сиб. гос. аэрокосмич. ун-т. - Красноярск, 2010. - 220 с.

14. Орлов А. И. Непараметрическое оценивание характеристик распределений вероятностей // Политематический сетевой эл. науч. журнал КГАУ. - Вып. -2015.- С. 1-20. 
15. Воскобойников Ю.Е., Тимошенко Е.И. Математическая статистика (с примерами в Excel) // уч. пособие. Новосибирск: НГАСУ (Сибстрин), 2006. - 152 с.

16. Газарян А.Ш., Мокриков С.С., Точечные оценки параметров распределения // Междунар. студ. науч. Вестник - Вып.3 (часть 1) - 2018 - С. 56-58.

17. Амридон Г.Б., Барлиани И.Я. Оценка неравноточно измеренных пространственных данных, полученных методом псевдонормальной оптимизации, и их свойства // ВестникСГУГиТ. -Том-22-№-4 2017 год с - 27-39.

18. Куштин И.Ф. Геодезия: обработка результатов измерений // учеб. пособие. - Ростов на Дону - 2006. С - 288.

19. Радионова М.В. Построение оптимальных доверительных интервалов для параметров положения и масштаба распределений // ГОУ ВПО - Пермский государственный университет, г. Пермь. - 2010. - С-7.

20. Попов В. А., Бренерман М. Х. Руководство к решению задач по теории вероятностей и математической статистике // Издательство Казанского государственного университета. 2008. - C-119.

(C) Н. Н. Кобелева, 3. Б. Бексултанова, 2021 